\title{
Prophylactic Hypogastric Artery Ligation during Placenta Percreta Surgery: A Retrospective Cohort Study
}

\author{
Theresa Kuhn, MD ${ }^{1}$ Kristina Martimucci, DO ${ }^{1}$ Abdulla Al-Khan, MD ${ }^{2}$ Robyn Bilinski, MD² \\ Stacy Zamudio, $\mathrm{PhD}^{2}$ Jesus Alvarez-Perez, $\mathrm{MD}^{2}$ \\ ${ }^{1}$ Department of Obstetrics and Gynecology, Hackensack University \\ Medical Center, Hackensack, New Jersey \\ ${ }^{2}$ Department of Maternal Fetal Medicine, Hackensack University \\ Medical Center, Hackensack, New Jersey \\ Address for correspondence Theresa Kuhn, MD, Department of \\ Obstetrics and Gynecology, Hackensack University Medical Center, \\ 30 Prospect Avenue, Hackensack, NJ 07601 \\ (e-mail: tk435@njms.rutgers.edu).
}

Am J Perinatol Rep 2018;8:e142-e145.

\begin{abstract}
Keywords

- abnormally invasive placentation

- placenta percreta

- cesarean hysterectomy

- hemorrhage

- hypogastric artery ligation

Objective To evaluate if prophylactic hypogastric artery ligation (HAL) decreases surgical blood loss and blood products transfused.

Study Design This is a retrospective cohort study comparing patients with placenta percreta undergoing prophylactic HAL at the time of cesarean hysterectomy versus those who did not. Data were presented as means \pm standard deviations, proportions, or medians with interquartile ranges. Demographic and clinical data were compared in the groups using Student's $t$-test for normally distributed data or the Mann-Whitney $U$ test for nonnormally distributed data. Fisher's exact test was used for proportions and categorical variables. Data are reported as significant where $p$ was $<0.05$.

Results There were 26 patients included in the control group with no HAL and 11 patients included in the study group. Estimated blood loss for the study group was $1,000 \mathrm{~mL}$ versus $800 \mathrm{~mL}$ in the control. Units of PRCBs transfused were 4.5 units in the study group versus 2 units for the control group. None of these measures were found to be statistically significant.

Conclusion Our data suggest there was no benefit in the use of prophylactic HAL in decreasing surgical blood loss or amount of blood products transfused in patients who had a cesarean hysterectomy performed for placenta percreta.

Précis Prophylactic HAL does not decrease blood loss during surgery for placenta percreta.
\end{abstract}

Hypogastric artery ligation (HAL) has been a surgical technique utilized to reduce hemorrhage during pelvic and obstetrical surgeries. HAL has the potential of being a life-saving measure that has been used when other more common modalities fail. ${ }^{1}$ The technique has been used to reduce pelvic blood flow when intraoperative hemorrhage is anticipated. ${ }^{2}$ The theoretical physiological change that occurs after HAL is a decrease in pulse pressure transforming an arterial system into a venous system, which decreases blood flow and therefore blood loss. ${ }^{3}$ Fewer obstetricians and gynecologists are performing prophylactic HAL for intraoperative hemorrhage control due to lack of experience and training. ${ }^{4}$

The use of HAL may have complications such as incomplete ligation, ureteral injury, hypogastric vein injury, or continued bleeding secondary to collateral circulation. ${ }^{5}$ There have been studies done on the use of prophylactic received

October 12, 2017

accepted after revision

March 22, 2018
DOI https://doi.org/

10.1055/s-0038-1666793. ISSN 2157-6998.
Copyright $\odot 2018$ by Thieme Medical

Publishers, Inc., 333 Seventh Avenue, New York, NY 10001, USA. Tel: +1(212) 584-4662.
License terms

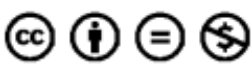


HAL, including a prospective trial that evaluated HAL at the time of radical hysterectomy and lymphadenectomy in gynecologic oncology patients. The trial found no significant decrease in surgical blood loss. ${ }^{6}$

Abnormally invasive placentation (AIP), such as placenta accreta, increta, and percreta, occurs secondary to uncontrolled angiogenesis of trophoblastic tissue of the placenta invading through the decidua, into the uterine myometrium, and possibly to adjacent structures. Invasive placentation can induce vascular remodeling of myometrial vessels, leading to significant hemorrhage if removal is attempted. ${ }^{7}$ The risk of maternal morbidity and mortality is high, especially in patients with placenta percreta. Morbidities include massive hemorrhage, maternal morbidity of a cesarean hysterectomy, blood transfusion, abdominal organ injury, mechanical ventilation, and intensive care unit admission. ${ }^{8}$ The most common treatment modality for patients with AIP is cesarean hysterectomy with or without HAL. ${ }^{9,10}$ The pelvis has extensive collateral blood flow, which can prevent adequate control of hemorrhage even after HAL. In a previous study by Clark et al, $42 \%$ of documented cases in which HAL was performed achieved adequate cessation of bleeding; however, only 1 out of 19 of these patients had AIP. ${ }^{11}$ To our knowledge, there are no data available that analyze HAL for obstetrical patients with AIP requiring cesarean hysterectomy.

The purpose of our study is to evaluate the effect of prophylactic HAL in decreasing total blood loss and amount of blood products transfused at the time of a cesarean hysterectomy for placenta percreta.

\section{Materials and Methods}

Our study is a retrospective cohort study in which all patients included were evaluated and treated at the Center for Abnormal Placentation at Hackensack University Medical Center from 2003 to 2015 . All procedures were performed by the same team of surgeons who routinely performed these cases, utilizing the same technique and protocol for each procedure. This is an institutional review boardapproved study, Pro00001951. Informed consent and ethics approval were obtained.

All patients with a preoperative diagnosis of placenta percreta suspected by ultrasound and magnetic resonance imaging (MRI) who underwent a cesarean hysterectomy and had a histopathological diagnosis of placenta percreta were included in this study.

All patients for whom the final histopathology was not a placenta percreta were excluded from this study. This was done to create a homogenous sample, making these results more generalizable to surgeons planning their percreta surgeries. We also excluded surgically staged procedures, in which HAL was purposefully not performed in preparation for embolization to the site of AIP and hysterectomy in a separate surgical procedure. The study group was composed of all patients who met the inclusion criteria and had an HAL during the time of cesarean hysterectomy. All HALs were performed bilaterally after the cesarean delivery and before hysterectomy for prophylaxis in anticipation of further blood loss. The control group was composed of the patients who met the inclusion criteria and did not undergo prophylactic HAL at the time of cesarean hysterectomy. A description of our multidisciplinary team and our surgical protocol has previously been described. ${ }^{10}$

For all the patients who met the inclusion criteria for this study, hospital admission data including operative reports were assessed. The data collected included maternal demographics, abnormal placentation known risk factors, and intraoperative data, which included estimated blood loss (EBL) and number of packed red blood cells (PRBCs) received.

Data were presented as means \pm standard deviation (SD), proportions, or medians with interquartile ranges. Data were analyzed using GraphPad Prism (La Jolla, CA). Demographic and clinical data were compared in the no HAL versus HAL groups using Student's $t$-test for normally distributed data or the Mann-Whitney $U$ test for nonnormally distributed data. Fisher's exact test was used for proportions and categorical variables. Data are reported as significant where $p$ was $<0.05$.

\section{Results}

A total of 45 patients were identified as having a preoperative diagnosis of placenta percreta by ultrasound and MRI. The positive predictive value for the histopathological diagnosis of placenta percreta was $100 \%$ in this patient cohort. The diagnosis was made by the pathologist if the villi penetrated the uterine serosa. Eight patients were excluded due to having a second staged surgical procedure for completion of the hysterectomy. The control group included 26 patients with no HAL, and the study group included 11 patients who underwent prophylactic HAL.

The groups were compared with respect to age, gravity, parity, body mass index (BMI), and other known risk factors for abnormal placentation, which we demonstrated in - Table 1. The BMI in the study group was found to be significantly lower in comparison to the control group ( - Table 1 ). This was the only variable found to be statistically significant.

The intraoperative data are also shown in - Table 1. The average EBL for the study group was $1,000 \mathrm{~mL}$ and for the control group the average EBL was $800 \mathrm{~mL}$. The average PRBC units transfused were 4.5 units for the study group and 2 units for the control group (-Table 1). Neither of these measures was found to be statistically significant.

\section{Discussion}

Our data suggest there is no benefit in the use of prophylactic HAL to decrease surgical blood loss or the amount of blood products transfused in patients having a cesarean hysterectomy performed for placenta percreta. This finding is similar to previous studies that looked at prophylactic HAL to decrease surgical blood loss during gynecologic oncology procedures. ${ }^{1,4,6}$ The use of HAL has been done prophylactically in other pelvic surgeries due to the potential decrease in pulse pressure limiting the pelvic blood flow; however, its use has not been analyzed for patients with a preoperative diagnosis of placenta percreta. ${ }^{3}$ 
e144 Prophylactic HAL during Placenta Percreta Surgery Kuhn et al.

Table 1 Maternal characteristics, clinical data, and abnormal placentation risk factors expressed as means \pm SD, median [IQR], proportions, and (range)

\begin{tabular}{|c|c|c|c|}
\hline & No HAL $(n=26)$ & $\operatorname{HAL}(n=11)$ & p-Value \\
\hline \multicolumn{4}{|l|}{ Maternal demographics } \\
\hline Age $(y)$ & $36 \pm 5(26-45)$ & $34 \pm 6(25-41)$ & 0.19 \\
\hline Gravidity & $6 \pm 3(2-14)$ & $5 \pm 2(3-9)$ & 0.42 \\
\hline Parity & $3[1,4](1-10)$ & $2[1,3](0-3)$ & 0.34 \\
\hline Prepregnancy BMI & $28.5 \pm 6.6(18.0-42.1)$ & $22.7 \pm 4.9(17.7-31.0)$ & $<0.05$ \\
\hline BMI at delivery & $31.7 \pm 6.5(21.0-49.0)$ & $26.9 \pm 5.6(21.0-37.0)$ & $<0.05$ \\
\hline \multicolumn{4}{|l|}{ Abnormal placentation risk factors } \\
\hline Advanced maternal age ( $\geq 35 \mathrm{y})$ & $62 \%$ & $45 \%$ & 0.48 \\
\hline IVF/ART & $4 \%$ & $0 \%$ & \\
\hline Prior cesarean delivery & $100 \%(1-5)$ & $100 \%(1-5)$ & 1.00 \\
\hline 1 & $27 \%$ & $18 \%$ & \\
\hline 2 & $35 \%$ & $18 \%$ & \\
\hline$\geq 3$ & $38 \%$ & $64 \%$ & \\
\hline Placenta previa & $77 \%$ & $82 \%$ & 1.00 \\
\hline \multicolumn{4}{|l|}{ Intraoperative data } \\
\hline Presurgical Hgb (g/dL) & $11.4 \pm 1.0(9.6-13.5)$ & $11.1 \pm 1.0(9.8-12.6)$ & 0.52 \\
\hline Postsurgical Hgb (g/dL) & $10.7 \pm 1.3(7.4-13.0)$ & $11.0 \pm 1.7(7.9-13.2)$ & 0.62 \\
\hline $\begin{array}{l}\text { Delta Hgb presurgery- } \\
\text { postsurgery values }(\mathrm{g} / \mathrm{dL})\end{array}$ & $-0.8 \pm 1.5(-5.4$ to 2.8$)$ & $\begin{array}{l}-0.2 \pm 2.0 \\
(-4.7 \text { to } 3.4)\end{array}$ & 0.41 \\
\hline $\mathrm{EBL}(\mathrm{mL})$ & $800[600,1,050](300-2,500)$ & $1,000[800,2,000](400-4,000)$ & 0.14 \\
\hline PRBCs administered intraoperatively ${ }^{a}$ & $2.0[1,4](1-8)$ & $4.5[2,6](2-8)$ & 0.08 \\
\hline Surgical time & $4.8[3,6](3-6)$ & $4.8[4,5](4-6)$ & 0.79 \\
\hline
\end{tabular}

Abbreviations: ART, assisted reproductive treatment; BMI, body mass index; EBL, estimated blood loss; Hgb, hemoglobin; IVF, in vitro fertilization; PRBCs, packed red blood cells.

${ }^{\mathrm{a}}$ Analysis limited to women who received transfusions (54\%, $n=14 / 26$ patients in the no HAL group; $72 \%, n=8 / 11$ in the HAL group, $p=0.46$ ). Postpartum PRBCs were administered to 2 ( $8 \%, 2$ units, 1 unit) of the no HAL group and 1 ( $9 \%, 1$ unit) in the HAL group $(p=0.21)$.

AIP is a unique surgical case compared with other gynecologic procedures. The gravid uterus, especially one with abnormal placentation, causes both a physiologic and pathological increase in large-diameter collateral blood vessels with the potential to hemorrhage. This can occur despite ligation of the hypogastric artery. For this reason, placenta percreta cases are known to have a risk for massive postpartum hemorrhage and maternal morbidity and mortality. ${ }^{2}$

HAL technique may have surgical complications, which include ureteral damage, perforation of the internal iliac vein, damage to the hypogastric nerve plexus, and buttock claudication. ${ }^{5}$ For this reason, it is imperative to assess if the use of prophylactic HAL is beneficial in decreasing maternal morbidity and mortality associated with the massive hemorrhage during placenta percreta surgery. Fortunately, we encountered none of these adverse events.

In our study, we did not identify a statistically significant difference in the total EBL between the control and study group. A limitation of the study may be that the blood loss was estimated and not quantified. However, the findings are supported by a similar change in preoperative and postoperative hemoglobin levels between both groups (see - Table 1).

Another important variable analyzed associated with surgical blood loss is number of PRBC units transfused. The number of PRBC units transfused in the study group, albeit higher, is not statistically significant. This finding may be explained by the small number of patients in the study group. Two patients out of the 11 total patients in the study group had a massive postpartum hemorrhage exceeding $3,000 \mathrm{~mL}$. In this small cohort of study patients, it is expected to see that these events may influence the results. Another limitation of our study is its retrospective design. The decision to perform an HAL was made intraoperatively by the surgeon. The decision to use prophylactic HAL may have been biased by the patient's anatomy and ability of the procedure to be completed bilaterally, abnormal placentation complexity, extent of invasion, and BMI of the patient. Patients who have severe abnormal placentation in which the surgeon believed that the completion of the surgery would be safer through a second staged procedure did not have an HAL so that embolization could be performed. Hence, the severity of 
the abnormal placentation may not be a main factor influencing the decision toward HAL, and the most complex cases did not receive HAL.

We also noted that the BMI was higher in the control group. Having a higher BMI may present a surgical challenge when performing an HAL and this finding could also be a potential selection bias. Finally, the sample size for both groups was small, limiting the ability to draw large conclusions.

We have previously identified and published the main factors that helped to decrease surgical blood loss in these cases, which included an intraoperative multidisciplinary approach and the learning curve of the surgeon. ${ }^{10}$

The incidence of AIP is low and the ideal study to answer the question if prophylactic HAL is of value at the time of placenta percreta surgery should be a larger prospective randomized trial.

Some research has been done to look at perioperative hypogastric artery balloon occlusion during gynecologic oncology procedures, as well as surgery for abnormal placentation. Although promising, further research is needed to see if this is a beneficial alternative. ${ }^{2,5,10,12-14}$ This modality overall has minimal procedure-related risks, but there have been reports of buttock claudication and lower extremity weakness. ${ }^{2}$ This may potentially be a better option than prophylactic HAL since the hypogastric artery is not surgically occluded and embolization of the pelvic vessels may be performed if a decision of a staged surgery is made.

\section{Disclosure}

This is original research from Hackensack University Medical Center, Maternal Fetal Medicine Department. Dr. Kuhn, Dr. Martimucci, Dr. Al-Khan, Dr. Bilinksi, Dr. Zamudio and Dr. Alvarez Perez were active participants and we wish to submit this research to the American Journal of Perinatology Reports. This research is not under consideration for publication in another journal. The authors report no biomedical financial interest or potential conflicts of interest.

\section{Conflict of Interest}

None.

\section{References}

1 Paraskevaides E, Noelke L, Afrasiabi M. Internal iliac artery ligation (IIAL) in obstetrics and gynaecology. Eur J Obstet Gynecol Reprod Biol 1993;52(01):73-75

2 Kidney DD, Nguyen AM, Ahdoot D, Bickmore D, Deutsch LS, Majors C. Prophylactic perioperative hypogastric artery balloon occlusion in abnormal placentation. AJR Am J Roentgenol 2001; 176(06):1521-1524

3 Burchell RC. Physiology of internal iliac artery ligation. J Obstet Gynaecol Br Commonw 1968;75(06):642-651

4 Papp Z, Tóth-Pál E, Papp C, et al. Hypogastric artery ligation for intractable pelvic hemorrhage. Int J Gynaecol Obstet 2006;92(01): 27-31

5 Fargeaudou Y, Morel O, Soyer P, et al. Persistent postpartum hemorrhage after failed arterial ligation: value of pelvic embolisation. Eur Radiol 2010;207(07):1777-1785

6 Arango HA, Hoffman MS, Roberts WS, et al. Does ligation of the hypogastric artery at the time of radical hysterectomy and lymphadenectomy decrease blood loss? Results of a prospective randomized trial. Int J Gynecol Cancer 1999;9(02):137-140

7 Al-Khan A, Gupta V, Illsley NP, et al. Maternal and fetal outcomes in placenta accreta after institution of team-managed care. Reprod Sci 2014;21(06):761-771

8 Upson K, Silver RM, Greene R, Lutomski J, Holt VL. Placenta accreta and maternal morbidity in the Republic of Ireland, 2005-2010. J Matern Fetal Neonatal Med 2014;27(01):24-29

9 Meehan FP, Casey C, Costello JN, Connolly CE. Placenta previa percreta with bladder involvement. Obstet Gynecol Surv 1989;44 (12):835-840

10 Hansch E, Chitkara U, McAlpine J, El-Sayed Y, Dake MD, Razavi MK. Pelvic arterial embolization for control of obstetric hemorrhage: a five-year experience. Am J Obstet Gynecol 1999;180 (6, Pt 1):1454-1460

11 Clark SL, Phelan JP, Yeh SY, Bruce SR, Paul RH. Hypogastric artery ligation for obstetric hemorrhage. Obstet Gynecol 1985;66(03): 353-356

12 Salim R, Chulski A, Romano S, Garmi G, Rudin M, Shalev E. Precesarean prophylactic balloon catheters for suspected placenta accreta: a randomized controlled trial. Obstet Gynecol 2015;126(05):1022-1028

13 Mei Y, Luo D, Lin Y. Clinical application of prophylactic internal iliac artery balloon occlusion combined with uterine artery embolization in patients with abnormally invasive placenta. J Matern Fetal Neonatal Med 2017;30:1-6

14 Cali G, Forlani F, Giambanco L, et al. Prophylactic use of intravascular balloon catheters in women with placenta accreta, increta and percreta. Eur J Obstet Gynecol Reprod Biol 2014;179:36-41 